\title{
BOLOMETRIC LIGHT CURVES OF SYMBIOTIC NOVAE
}

\author{
U. MÜRSET and H. NUSSBAUMER \\ Institute of Astronomy \\ ETH-Zentrum \\ CH-8092 Zürich \\ Switzerland
}

SUMMARY. We determine bolometric light curves and total energies radiated away during the outburst of symbiotic novae. Time integrated lower limits to the total energy of $0.9 \times 10^{46}<\mathrm{E}[\mathrm{erg}]<7 \times 10^{46}$ are found. Thus, the output is comparable to, or larger than the total energy production of a classical nova outburst. From the mass-luminosity relation we find the underlying stellar masses to be $0.5<M / M_{\odot}<1.1$.

\section{Symbiotic novae}

Symbiotic novae differ from classical novae by the slowness and long duration of the outburst. They occur - in contrast to classical novae - in relatively widely separated binary systems, the accretion is therefore due to wind accretion, without the formation of accretion disks.

There are seven reasonably observed symbiotic novae: AG Peg, RT Ser, RR Tel, V1016 Cyg, HBV 475, HM Sge, and PU Vul. None of the seven systems has run the full course of an outburst, we can therefore only determine lower limits to the total energy outputs.

In a symbiotic nova the rise to maximum luminosity takes typically a few weeks or months. At the end of that rise to high luminosity the object either enters a 'supergiant' phase, in which it remains for a few years before entering the nebular phase, or it progresses straight into the nebular phase. The supergiant phase corresponds to an expanded object which emits mostly an F- or A-supergiant spectrum. AG Peg, RR Tel, RT Ser and PU Vul have spent extended periods in the supergiant phase, whereas V1016 Cyg, HBV 475, and HM Sge did not. In the nebular phase we find for the outbursting object typical effective temperatures, $T$, between $50000 \mathrm{~K}$ and $200000 \mathrm{~K}$. A symbiotic nova remains in the nebular phase for many dozens of years, its decline to pre-outburst luminosity is a very slow process. Mürset et al. (1991, thereafter MNSV) have determined $T$ and luminosities, $L$, for a number of symbiotic stars.

In symbiotic novae the cool companion provides a nebula which converts EUV photons through ionization and recombination into observable photons. We have therefore a much sounder basis for luminosity determinations than is available for classical novae.

\section{A relation between luminosity and apparent visual brightness}

For determining luminosities we rely on IUE observations and published U, B, and V magnitudes. The luminosity $L$ of the ionizing source is related to the observed magnitudes $m_{\Lambda}^{\text {obs }}(\Lambda=U, B$, or V) through 


$$
\frac{L}{L_{\odot}}=\left(\frac{d}{10 \mathrm{pc}}\right)^{2} \cdot\left(10^{-0.4 \cdot m_{\Lambda}^{\mathrm{obs}}}-10^{-0.4 \cdot m_{\Lambda}^{\mathrm{cool}}}\right) \cdot 10^{0.4 \cdot\left(\mathrm{B} \cdot \mathrm{C} \cdot \Lambda+A_{\Lambda}+\mathrm{M}_{\mathrm{bol}}^{\odot}\right)},
$$

where $m_{\Lambda}^{\text {cool }}$ is the respective apparent magnitude of the cool component, B.C. $\Lambda=m_{\Lambda}-m_{\text {bol }}$ the bolometric correction, $d$ the distance, and $A_{\Lambda}$ the interstellar extinction.

If the ionizing radiation is black body radiation and the nebula is radiation bounded, B.C. $\Lambda$ depends only on $T$. The B.C.( $T$ )-function goes through a flat minimum at $T \approx 80000 \mathrm{~K}$ and varies only slightly in the range of interest. For the supergiant phase we assume the spectral type to be an appropriate indicator of $T$, and we calculate $L$ by applying the bolometric corrections for normal stars of the corresponding spectral type. - The contributions of the cool components to the observed magnitudes are derived from measurements before outburst or during eclipse, or from infrared observations. In other cases the optical spectrum shows no trace of a cool component, so that it can be neglected.

For objects observed with IUE, the UV spectra allow to distinguish between radiation bounded and particle bounded nebulae. That work has already been carried out by MNSV, and we adopt their $L$ values.

For determining luminosities outside the IUE period, we require $d, E_{\mathrm{B}-\mathrm{V}}, m_{\Lambda}$, B.C..$_{\Lambda}$ and the spectral types during the supergiant phase. They are taken from the literature. In pre-IUE observations we cannot distinguish between particle bounded and radiation bounded nebulae. In those symbiotic novae which are are partly density bounded (MNSV) this leads to an underestimate of $L$.

\section{Results for individual objects}

AG Pegasi. AG Peg may be close to the end of its outburst, which began in the middle of the $19^{\text {th }}$ century. AG Peg is the system with the slowest evolution. It is also the system with the smallest peak luminosity, and the integrated energy is relatively low. The luminosity during the first few decades is uncertain, due to lack of spectral information. The $1905-1921$ minimum is defined by observations in 1905, 1921, and 1907-08 where Lundmark (1921) gives '1907-08 $<8^{\mathrm{m}} \cdot 1$ ', and comments that this low magnitude should be taken as a sign of variability.

RT Serpentis. Payne-Gaposchkin and Gaposchkin (1938) report the early light curve. The outburst became noticeable in 1909. There was a steep rise from below $16^{\mathrm{m}}$ to $11^{\mathrm{m}} \cdot 3$, which apparently occurred within 40 days, this is unusually fast for a symbiotic nova. From 1919 to 1921 it slowly changed into the nebular phase. The continuum of the 1986 spectrum is dominated by contributions of the cool star. The system has probably returned to pre-outburst brightness. Like AG Peg, RT Ser is a slow system. It is also similar to AG Peg in its low luminosity and total energy output. However, for this system the distance is rather uncertain and $E_{\mathrm{B}-\mathrm{v}}=0$ was adopted because of lack of any reliable estimates; this can easily result in a considerable underestimate of the absolute luminosity.

RR Telescopii. RR Tel has a reasonably well documented outburst history. The outburst itself can be well divided into a supergiant and a nebular phase. It began in 1944 when it went into the supergiant phase. In the autumn of 1949 a rapid transition led to the nebular phase. In $1991 \mathrm{RR}$ Tel is still in the nebular state with a radiation temperature of $T \approx 140000 \mathrm{~K}$. MNSV find RR Tel to be particle bounded. $L$ may therefore be underestimated in the years preceeding IUE observations.

V1016 Cygni. The outburst of V1016 Cyg began in 1963. It did not pass through an extended giant phase, but developed a nebular spectrum practically at the end of the rise to maximum in 1965 . From the IUE spectra MNSV find a mean radiation temperature of $T \approx 135000 \mathrm{~K}$ and evidence for a partly particle 

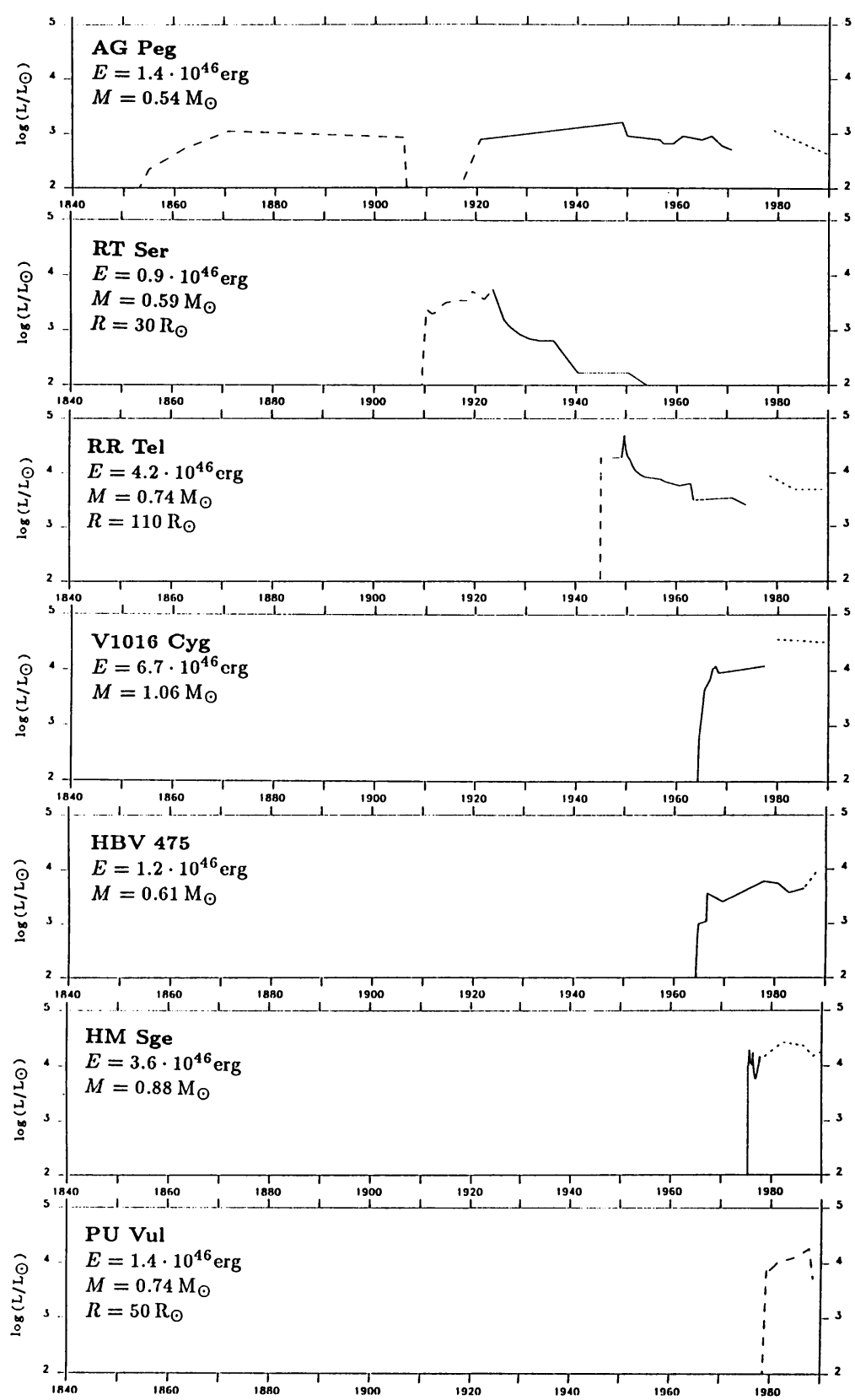

Bolometric light curves of symbiotic novae. Dashed: $L$ during supergiant phase; full lines: nebular phase, as derived from photometric data; dotted: nebular phase, as derived from IUE spectra (after MNSV). Discontinuities between full and dotted lines are probably due to the assumption of a radiation bounded nebula in the derivation of $L$ from visual photometry. Also given are the integrated energies $E$, the masses $M$ according to the core-mass - luminosity relation, and the radii $R$ during the supergiant phase. 
bounded nebula, which may imply an underestimate of $L$ in the pre-IUE period. With $7 \times 10^{46}$ erg V1016 Cyg is the symbiotic nova with the highest integrated radiation output up to 1991.

HBV 475 ( = V1329 Cygni). In 1964 HBV 475 accelerated its brightening to reach maximum in 1966. Since then its brightness has been slowly decreasing, at the time of the last IUE observation in 1989 it has reached $\approx 13^{\mathrm{m}}$. MNSV find HBV 475 to be radiation bounded. In accordance with Nussbaumer and Vogel (1991) we assume the periodic attenuation of the observable flux to be due to eclipse by neutral gas. The real luminosity of the hot source therefore corresponds to maximum luminosity.

HM Sagittae. The outburst history of HM Sge has been studied by Nussbaumer and Vogel (1990). It began in 1975 . The 1976 spectra indicate $T \approx 40000 \mathrm{~K}$. The temperature then was continuously increasing and has in 1990 reached a value of close to $200000 \mathrm{~K}$.

PU Vulpeculae. PU Vul is the youngest symbiotic nova. Its outburst began in late 1977, its luminosity reached a maximum in 1979. The 1979 - 1982 spectra show that the system consists of an $M$ giant and an object emitting the spectrum of an F supergiant. The spectrum gradually turned hotter, and by 1989 had become that of an early A type giant. The object seems to have entered the nebular phase in 1990.

\section{Discussion and comparison with classical novae}

Available observations suggest for classical novae $10^{46}-10^{47} \mathrm{erg}$ as total energy output. However, it is difficult to determine absolute luminosities of classical novae, since in most cases their distances are badly known. For two novae where distances are well known, LMC 1988 \#1 and LMC 1988 \#2, we extract from Austin et al. (1990, Fig. 2) for the first 200 days of their outburst total energies of $2 \times 10^{45}$ erg. Our results for symbiotic novae give total time integrated energies of $0.9 \times 10^{46} \lesssim E[\mathrm{erg}] \lesssim 6 \times 10^{46}$. This is superior to the two LMC novae. Our total energy corresponds to an equivalent of $8 \times 10^{-7}$ to $5 \times 10^{-6} M_{\odot}$ of hydrogen converted into helium.

Model calculations for nova outbursts predict a phase of constant luminosity following visual maximum. Considering uncertainties in the observations, our light curves seem to confirm this in first order. We apply the core-mass - luminosity relation $L / L_{\odot}=59250 \cdot\left(M / M_{\odot}-0.522\right)$ for post-AGB stars (Paczyniski 1971). The results are given in the Figure. - We also give the radii of the photospheres during the supergiant phase. They are much smaller than the binary separation.

A detailed description of the work presented here will be submitted for publication in Astronomy and Astrophysics (Mürset and Nussbaumer, 1991).

\section{References:}

Austin, S., Starrfield, S., Saizar, P., Shore, S.N., Sonneborn, G.: 1990, in Evolution in Astrophysics, ESA SP-310, p. 367

Lundmark, K.: 1921, Astronomische Nachrichten, 213, 94

Mürset, U., Nussbaumer, H.: 1991, Astron. Astrophys. (in preparation)

Mürset, U., Nussbaumer, H., Schmid, H.M., Vogel, M.: 1991, Astron. Astrophys. (in press), (MNSV)

Nussbaumer, H.: 1991, in IAU Symposium No. 151, (this volume)

Nussbaumer, H., Vogel, M.: 1990, Astron. Astrophys. 236, 117

Nussbaumer, H., Vogel, M.: 1991, Astron. Astrophys. (in press)

Paczyński, B.: 1971, Acta Astr. 21, 417

Payne-Gaposchkin, C., Gaposchkin, S.: 1938, Variable Stars, Harvard Obs. Monograph No. 5, p. 263 